\title{
Amplitude and frequency changes in surface EMG of biceps femoris during five days Bruce Protocol treadmill test
}

\begin{abstract}
Electromyography (EMG) is one of the indirect tools in indexing fatigue. Fatigue can be detected when there are changes on amplitude and frequency. However, various outcomes from literature make researchers conclude that EMG is not a reliable tool to measure fatigue. This paper investigates EMG behavior of biceps femoris in median frequency and mean absolute value during five days of Bruce Protocol treadmill test. Before that, surface EMG signals are filtered using band pass filter cut-off at $20-500 \mathrm{~Hz}$ and are de-noised using db45 1 decimated wavelet transform. Five participants achieved more than $85 \%$ of their maximal heart rate during the running activity. The authors also consider other markers of fatigue such as performance, muscle soreness and lethargy as indicators to adaptation and maladaptation conditions. Result shows that turning points of median frequency and mean absolute value are very significant in indexing fatigue and indicators to adaptation of resistive training.
\end{abstract}

Keyword: EMG; Biceps femoris; Bruce Protocol treadmill test; Fatigue 